\title{
Écophysiologie de la maturation sexuelle et de la ponte de l'huître creuse Crassostrea gigas : réponses métaboliques (respiration) et alimentaires (filtration, absorption) en fonction des différents stades de maturation
}

\author{
Patrick Soletchnik, Daniel Razet, Philippe Geairon, \\ Nicole Faury et Philippe Goulletquer \\ IFREMER, GAP. Unité de Recherche Aquacole de Poitou Charenle, Mus de Loup, \\ BP 133, F-17390 La Tremblade, Franse. E-mail: patrick.soletchnik@ifremer.fr
}

Reçu le 3 octobre 1996; accepté le 11 février 1997.

Soletchnik P., D. Razet, P. Geairon, N. Faury, P. Goulletquer. Aquat. Living Resour., 1997, 10, 177-185.

Ecophysiology of maturation and spawning in oyster (Crassostrea gigas): Metabolic (respiration) and feeding (clearance and absorption rates) responses at different maturation stages.

Abstract

The study of maturation and spawning of the oyster is part of a research program to investigate the summer mortalities of the oysters, Crassostrea gigas in Marennes-Oléron Bay. Four maturity stages were simultaneously obtained by diet and thermal conditioning (immature, low maturation, mature and post-spawning stages). Measurements of clearance, filtration, absorption and respiration rates allowed a calculation of the scope for growth and hence an estimation of the oyster's energetic budget at various maturity stages. Male and female oysters had similar physiological responses. The filtration rate ranged from 2.4 to $2.61 . \mathrm{h}^{-1}$ at the early stages of maturation and decreased to $1.81 . \mathrm{h}^{-1}$ during the maturity stage. Growth rate resulting from gonad development did not induce filtration rate changes. Mature 2.5 and 1.5-year-old oysters showed a negative energy budget reaching -15 and $-90 \mathrm{~J}^{-1} \mathrm{~h}^{-1}$ respectively. By contrast, non-ripe oysters had scope for growth in the range 110 to $170 \mathrm{~J} \cdot \mathrm{h}^{-1}$. A negative energy budget during the high maturation stage resulted from a reduced absorption efficiency. A new allometric relationship for the respiration model of $C$. gigas was defined during vitellogenesis with a 0.574 coefficient value. Based on our results, the oyster's physiological weakness during vitellogenesis should be considered as a part of explanation for spring and summer mortalities of cultured oysters in Marennes-Oléron Bay.

Keywords: Oyster, Crassostrea gigas, conditioning, maturation, filtration, absorption and respiration rates.

Résumé

L'étude de la maturation et de la ponte de l'huître creuse Crassostrea gigas, s'intègre dans une large problématique visant à comprendre les causes de mortalité qui surviennent épisodiquement dans le bassin de Marennes-Oléron au printemps et en été. Un conditionnement thermique et alimentaire des élevages permet d'obtenir simultanément des huîtres à tous les stades de maturité sexuelle. Le calcul des taux de filtration, consommation, absorption et respiration permet d'estimer le potentiel de croissance, et donne le bilan énergétique des huîtres aux différents stades sexuels. Les réponses physiologiques sont semblables pour des huîtres mâles ou femelles. La filtration de 2,4 à $2,61 . h^{-1}$, passe à $1,81 \cdot h^{-1}$ à un stade de maturation avancée. La prise de poids liée au développement des gonades ne semble pas avoir d'effet sur le taux de filtration. Les huîtres de 2,5 ans présentent un bilan énergétique déficitaire $\left(-15 \mathrm{~J} \cdot \mathrm{h}^{-1}\right)$ à un stade de maturation avancée. Ce déficit est confirmé $\left(-90 \mathrm{~J}^{-h^{-1}}\right)$ sur des huîtres de 1,5 ans au même stade de maturation. En revanche, les huîtres immatures, en début de maturation ou en post-ponte, ont un potentiel de croissance compris entre 110 et $170 \mathrm{~J}^{-h^{1}}$. Le déficit énergétique observé au stade de maturation avancée relève essentiellement de la fonction d'absorption qui chute de façon importante en période de forte gamétogenèse. L'étude de la respiration permet de définir une allométrie particulière à la croissance 
gonadique avec un coefficient d'allométrie de 0,574. La faiblesse physiologique des huîtres, observée à un stade de maturation avancée, doit être prise en compte dans la recherche des facteurs responsables des mortalités printanières et estivales affectant les cheptels en élevage dans le bassin de Marennes-(Oléron.

Mots-clés : Huître, Crassostrea gigas, conditionnement, maturation, filtration, absorption, respiration.

\section{INTRODUCTION}

Dans le bassin de Marennes-Oléron, premier bassin ostréicole européen, l'huitre creuse Crassostrea gigas connaît des épisodes de mortalités au printemps et en été, marqués en 1988 par la disparition de plusieurs milliers de tonnes sur le secteur du banc ostréicole de Ronce (Bodoy et al., 1990). Ces mortalités se rencontrent également dans d'autres bassins ostréicoles (Maurer et al., 1986). Les principales causes invoquées sont une évolution du «pool » génétique de $C$. gigas dans le bassin de Marennes-Oléron (liée à une pratique sélective de l'élevage), une surcharge en biomasse (pouvant être associée à une dérive des pratiques ostréicoles durant la dernière décennie), une détérioration progressive de l'environnement du bassin et/ou une conjonction de plusieurs des causes évoquées ci-dessus.

Les mortalités printanières se manifestent en période de maturation des huîtres. En absence de cause pathogène bien identifiéc, les conditions de température et l'état physiologique des huîtres en cours de maturation, font partie des facteurs suspectés. L'état et la réponse physiologique de l'huître sontils significativement marqués par son stade de maturation?

Les modifications biochimiques survenant au cours de la vitellogenèse des bivalves sont connues de longue date (Lubet, 1959; Gabbott, 1976). Perdue et al. (1981) ont mis en évidence la relation existant entre les cycles des constituants biochimiques et la gamétogenèse. Chez Crassostrea gigas, les changements saisonniers entre les compartiments somatiques et gonadiques ont été étudiés par de nombreux auteurs. Dans le bassin de Marennes-Oléron, le cycle de gamétogenèse est décrit par Deslous-Paoli et al. (1982) et DeslousPaoli et Héral (1988). Quelques auteurs traitent des clésordres physiologiques (Mori, 1979) ou de constitution biochimique (Berthomé et al., 1982) de C. gigas dans le milicu naturel. En laboratoire, grâce au contrôle de la température et des conditions trophiques, les huîtres sont amenées à pondre début mai, alors que les pontes naturelles de l'année 1995 ont commencé mi-août 1995. Les processus de vitellogenèse et la ponte sont artificiellement accélérés par des conditions d'élevage à température supérieure à celle des normales saisonnières (Price et Maurer, 1971).

Quelques études traitent du coût énergétique de la reproduction ou de la relation entre les stades de maturation et les réponses physiologiques (Thompson, 1984; Newell et Thompson, 1984).
Quelques travaux concernent la comparaison de triplö̈des et de diplö̈des de Crassosirea gigas en période de reproduction (Davis, 1988; Shpigel et al., 1992). Des observations faites dans le milieu naturel ont montré que les mortalités estivales affectaient souvent les jeunes huîtres au cours des deux premières années. Crassostrea gigas peut atteindre l'âge de première maturité sexuelle en 12-18 mois sous nos Jatitudes et parfois en moins d'un an (Dinamini, 1987). L'allocation d'énergie vers la croissance ou la maturation connaît une grande variabilité durant la première année.

Les études concernant l'écosystème conchylicole (Héral et al., 1983, Deslous-Paoli et al., 1987, 1992) ont conduit à développer un modèle biologique de croissance de l'huître creuse Crassostrea gigas, principale espèce élevée dans le bassin de MarennesOléron (Bacher, 1989; Raillard, 1991; Bougrier et al., 1995; Barillé et al., 1997). Ce modèle est élaboré à partir de mesures expérimentales sur des huîtres adultes, en dehors de la période de maturation et de ponte. Les modèles de respiration et de filtration développés jusqu'à présent concernent exclusivement les huîtres en période de repos sexuel (Bougrier et $a l ., 1995)$. Le modèle biologique de croissance de Crassostrea gigas s'appuie sur ces deux modèles (Barillé et al., 1997).

L'objectif de cette étude est (1) de tester si le stade de maturation a une influence sur l'état physiologique de l'huître et (2) de compléter le modèle biologique en intégrant des données acquises en période de gamétogenèse et de ponte.

\section{MATÉRIEL ET MÉTHODES}

\section{Le matériel biologique}

Deux populations d'huîtres sont utilisées au cours de cette expérimentation. Une première population de 300 individus de 2,5 ans et 1,26 g de poids sec en début avril 1995 est issue d'un test zootechnique d'affinage conduit en claires ostréicoles entre octobre 1994 et janvier 1995 (lots 1, 2, 3 ct 5). Une deuxième population issue de captage naturel dans le bassin de Marennes-Oléron est constituée de 100 huîtres de « 18 mois» de $1,36 \mathrm{~g}$ de poids $\mathrm{sec}$ en début d'expérimentation (lot 4).

\section{Le conditionnement des huîtres}

L'aliment produit en bassins extérieurs est constitué de blooms naturels de la diatomée: Skeletonema costatum et du phytoflagellé : Tetraselmis suecica. 
L'induction et le contrôle de la maturation sont réalisés grâce au facteur de température en déplaçant progressivement des huîtres issues d'une population homogène d'un bassin de stockage en condition "froide » vers des « raceways" de maturation en condition chaude. La Figure 1 présente les différents régimes de température permettant ainsi de définir les 5 lots d'huîtres. Chaque lot est constitué d'environ 75 huîtres. Le lot 1 est caractérisé par un conditionnement de longue duréc à température contrôlée de $20-23^{\circ} \mathrm{C}$, c'est-à-dire proche de $22^{\circ} \mathrm{C}$, idéale pour le conditionnement de C. gigas en écloserie (Lubet, 1991). Les lots 4 et 5 (2 classes d'âge) suivent les conditions thermiques du milieu naturel, variant progressivement de 13 à $22^{\circ} \mathrm{C}$. Les lots 2 et 3 subissent un conditionnement intermédiaire à ceux des lots 1 et 5 (Fig. 1).

\section{Mesures des fonctions physiologiques}

Le système expérimental décrit par Barillé et al. (1993) permet d'effectuer les mesures des principales fonctions physiologiques sur les mollusques filtreurs. L'aliment particulaire (algues de culture : Skeletonema costatum, Isochrysis galbana) transite d'un bac de stockage jusqu'à un bac d'homogénéisation, avant de parvenir, en flux continu, dans les cellules de mesures (Fig. 2).

Les biodépôts de l'huître, fèces et pseudo-fèces, sont collectés dans les cellules durant la période de mesures physiologiques (Barillé et al., 1993). Les concentrations particulaires sont mesurées en entréc et en sortie des cellules de mesures à partir des volumes d'éau collectés (Fig. 2, Tableau 1). Les méthodes d'analyses de la matière particulaire totale, organique et minérale déterminées après crémation à $450^{\circ} \mathrm{C}$, sont communes à l'eau et aux biodépôts. La méthode d'analyse est décrite par Razet et al. (1990). La mesure de respiration est effectuée dans une enceinte à volume constant de 0,5 à 1 litre en fonction du groupe d'âge (Fig. 3). La concentration d'oxygène est mesuréc toutes les 10 minutes grâce à une sonde oxymétrique équipée d'un agitateur magnétique.

Tableau 1. - Définition des notations utilisées.

Definition of the notations used.

\begin{tabular}{|c|c|}
\hline MOP & $\begin{array}{l}\left.\text { Matière organique particulaire (mg. } \mathrm{I}^{-1}\right) \\
\text { Particulate organic matter }\end{array}$ \\
\hline $\mathrm{MOP}_{\mathrm{e}}$ & $\begin{array}{l}\text { Matière organique particulaire en entrée des cellules } \\
\text { de mesure (mg. }{ }^{1} \text { ) } \\
\text { Particulate organic malter. Input }\end{array}$ \\
\hline MOPs & $\begin{array}{l}\text { Matière organique particulairc cn sortie de celules de } \\
\text { mesure (mg. } \mathrm{J}^{-1} \text { ) } \\
\text { Particulate organic matter. Output }\end{array}$ \\
\hline $\mathrm{MOP}_{\mathrm{pf}}$ & $\begin{array}{l}\text { Contenu organique dans les pseudo-feces }\left(\mathrm{mg}^{-1} \mathrm{~h}^{-1}\right) \\
\text { Organic production in pseudofeces }\end{array}$ \\
\hline $\mathrm{MOP}_{\mathrm{f}}$ & $\begin{array}{l}\text { Contenu organique dans les feces }\left(\mathrm{mg} \cdot \mathrm{h}^{-1}\right) \\
\text { Organic production feces }\end{array}$ \\
\hline d & $\begin{array}{l}\text { Débit }\left(1 . \mathrm{h}^{-1}\right) \\
\text { Flow rate }\end{array}$ \\
\hline
\end{tabular}
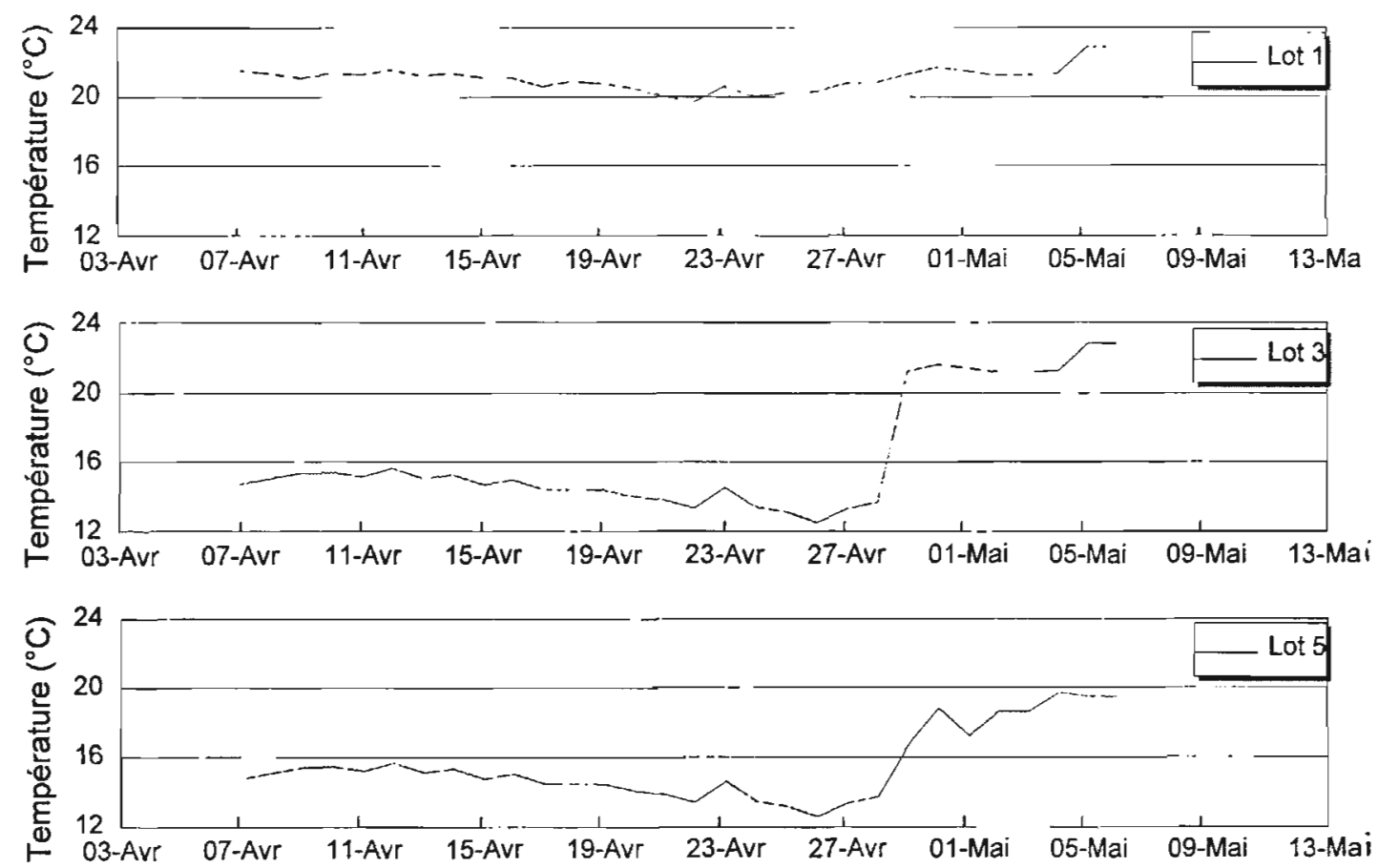

Figure 1. - Conditionnement thermique des différents lots d'buitres 1,3 et 5 .

Thermal conditioning for various batches of oysters $I, 3$ and 5 .

Aquat. Living Resour., Vol. 10, no $3-1997$ 
Sept cycles de mesures physiologiques sont réalisés sur chacune des 120 huîtres issues des lots conditionnés à des températures différentes entre le

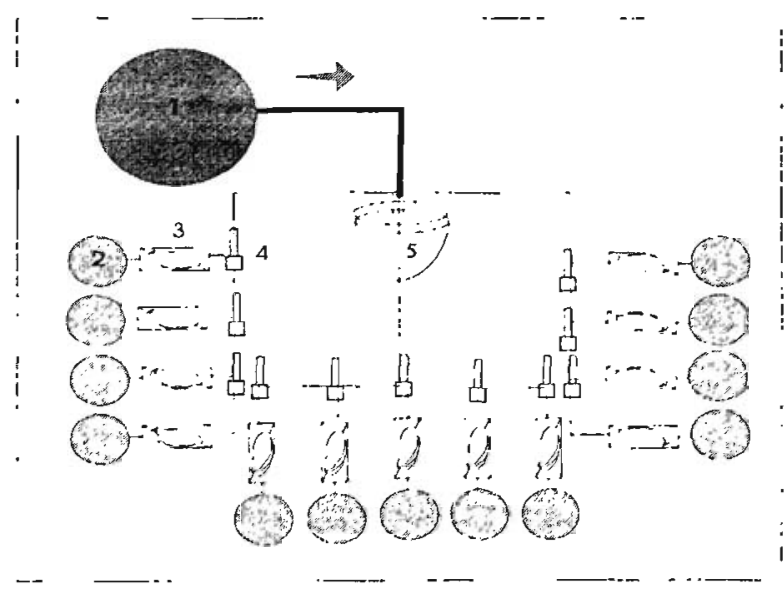

Figure 2. - Système expérimental utilisé pour relever Jes paramètres physiologiques de l'huittre Crassostrea gigas. I : Bac d'homogénéjsation de l"aliment. 2: Bac de collecte de l'eau en sortie des cellules de mesures physiologiques. 3 : Cellules de mesures physiologiques avec huitre vivante ou contróle sur coquille vide. 4 : débitmètre en entrée des cellules de mesures physiologiques. 5 : Réacteur de distrihution de l'eau de mer dans les cellules de mesures.

Experimental set-up for physiological measurements on Crassostrea gigas oyster. 1: Homogenizing tank for diet. 2: Collecting tank for outflow water from experimental chambers. 3: Experimental chamber set with live oyster and dead oyster as control. 4: flow-meter. 5: water supply tank.
4 mai et le 16 mai 1995 . Le choix d'utilisation d'huîtres de tel ou tel lot de conditionnement est guidé par le souci d'équilibrer au mieux les effectifs d'huîtres pour chaque stade sexuel déterminé a posteriori après les mesures physiologiques. La qualité de l'eau pendant les expérimentations est identique à celle du conditionnement des huîtres.

La fonction de filtration ( $1 . h^{1}$ ) est calculée selon la formule suivante (voir Tableau 1 pour les notations):

Filtration $=\mathrm{d} \times\left[\left(\mathrm{MOP}_{\mathrm{e}}-\mathrm{MOP}_{\mathrm{s}}\right) / \mathrm{MOP}_{\mathrm{e}}\right]$

L'absorption (mg.h $\left.h^{-1}\right)$ est calculée par :

Absorption $=\mathrm{d} \times\left[\left(\mathrm{MOP}_{\mathrm{e}}-\mathrm{MOP}_{\mathrm{s}}\right)\right]-\mathrm{MOP}_{\mathrm{pf}}-\mathrm{MOP}_{\mathrm{f}}$

Le potentiel de croissance $\left(\mathrm{J} \mathrm{h}^{-1}\right)$ est estimé par la formule suivante (Lucas, 1982) :

Potentiel de croissance $=$ Absorption - Respiration

La fonction de respiration représente la consommation d'oxygène ( $\mathrm{mg} \mathrm{O}_{2} \cdot \mathrm{h}^{\prime}$ ) convertie en $\mathrm{J} . \mathrm{h}^{-1}$ par le coeflicient de conversion énergétique de 0,45 J. $\mu \mathrm{mol}^{-1} \mathrm{O}_{2}$ (Bayne, 1976). Le coefficient de conversion énergétique de la matière organique

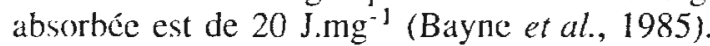

\section{Analyse des huîtres}

A l'issue de chacun des cycles de mesures physiologiques, pour chaque huître, le poids sec est déterminé après congélation de la chair fraîche, puis lyophilisation pendant 36 heures. Les analyses biochimiques sont celles décrites par Deslous-Paoli et Héral (1988) pour le dosage des protéines, lipides et glucides.

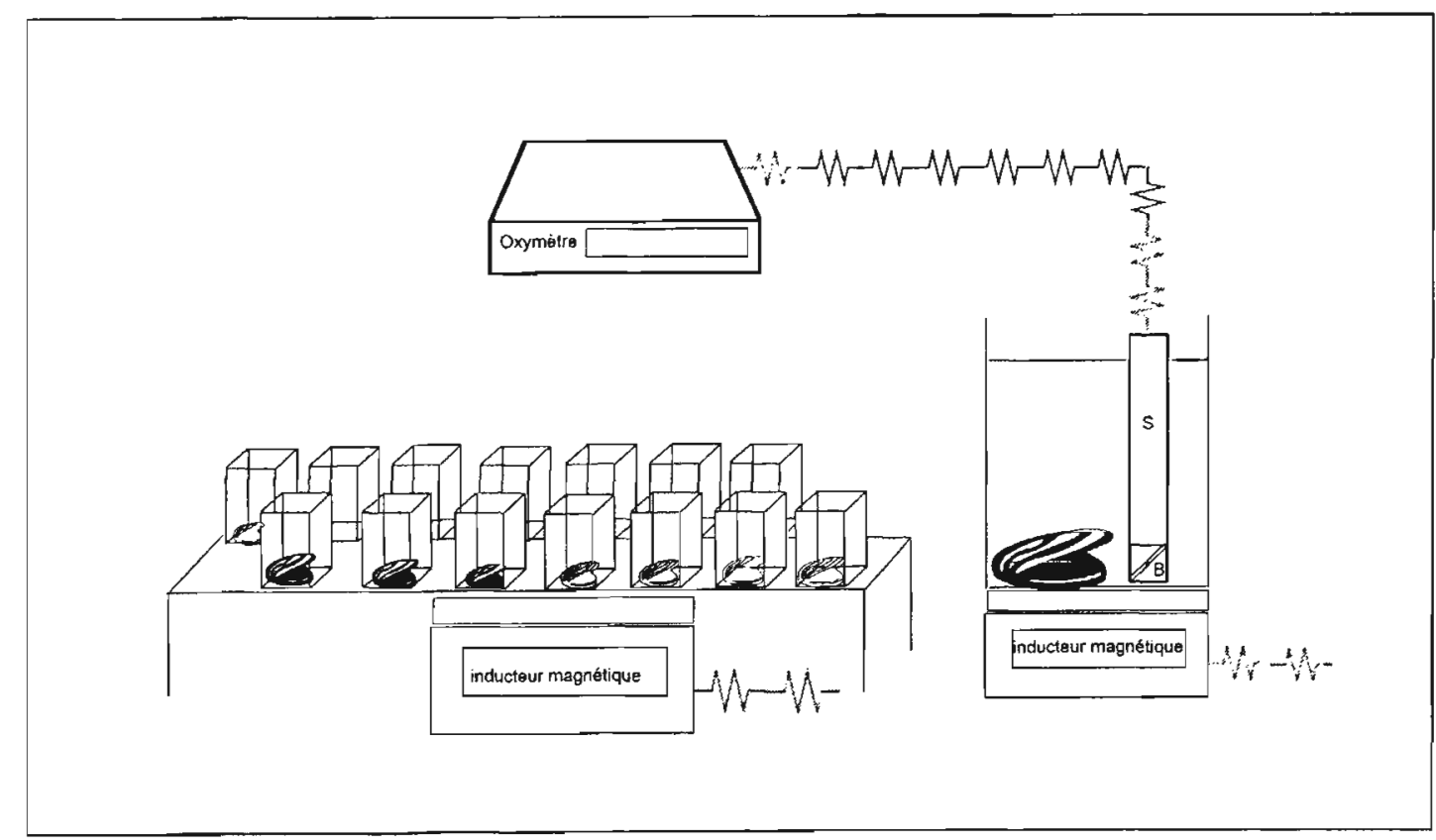

Figure 3. - Cellules de mesure de la respiration chez Crassostrea gigas. L'équipement est constitué d'une sonde oxygène (S) équipée à son extrémité d'un agitateur rotatif aimanté (B).

Experimental chamber for respiration measurement for Crassustrea gigas. An uxygen probe set with a rotating magnet is the main equipment. 
A l'issue des mesures physiologiques effectuées sur les huîtres des différents lots conditionnés par la température, les stades de maturation sexuelle sont déterminés sur la base d'observations macroscopiques et microscopiques à partir de critères issus des échelles de maturation de Marteil (1976) et Le Dantec (1968). Les critères macroscopiques concernent le recouvrement de la masse hépatopancréatique par la gonade et l'émission de produits génitaux à partir d'une pression sur la gonade. Après frottis des gonades, les critères microscopiques permettent de définir le sexe et précisent la mobilité des cellules sexuelles mâles, la taille et forme des ovocytes. La somme des observations macroscopiques et microscopiques pour mâles et femelles définit une échelle relative de maturité sexuelle comprise entre 0 et 2 à laquelle s'ajoute un stade 3 de post-ponte. L'échelle est la suivante :

0 : individus immatures

1 : stade de début de gamétogenèse

2 : stade de maturation avancée

3 : stade de post-ponte

Les huîtres au stade 3 sont obtenues au moyen d'un choc d'une amplitude thermique de $7^{\circ} \mathrm{C}\left(21\right.$ à $\left.28^{\circ} \mathrm{C}\right)$.

En terme de conditionnement, les huîtres du stade 3 (post-ponte) sont issues des lots 1 et 2 . Les huîtres du stade 2 (maturation avancée) proviennent des lots 3 et 4 ( 2 classes d'âge), et les huîtres en début de maturation sont issues pour l'essentiel du lot 5 .

\section{Analyse statistique}

Deux analyses de covariance (covariable $=$ poids sec) comparent les descripteurs de la chair (protéines, lipides et glucides) et les performances physiologiques de l'huître (filtration, respiration et potentiel de croissance) selon deux facteurs : le stade de maturité sexuelle et le sexe, pour la première analyse (avec la population d'huîtres de 2,5 ans); et l'âge (1,5 ans et 2,5 ans) et le sexe pour une deuxième analyse (avec les huîtres au stade $2 \mathrm{de} \mathrm{la} \mathrm{maturité} \mathrm{sexuelle).} \mathrm{Une}$ régression non linéaire permet de préciser la relation d'allométrie de la fonction de respiration et de filtration au cours de la maturation, soit aux stades 1 et 2 définis ci-dessus.

\section{RÉSULTATS}

\section{Comparaison selon les stades de maturité sexuelle et le sexe des huîtres}

Le poids sec des huîtres, au moment des mesures physiologiques, est compris entre 2,5 et $2,8 \mathrm{~g}$ quel que soit le stade de maturité sexuelle et quel que soit le sexe de l'huître (Tablcau 2). Une différence significative pour les lipides $(p=0,05)$ et les glucides $(p=0,01)$ apparait sclon le stade de maturité sexuelle avec une quantité relativement plus faible en lipides $(0,24 \mathrm{~g})$ el plus forte en glucides $(0,39 \mathrm{~g})$ dans la chair des huîtres au stade de post-ponte. Aucune différence n'apparaît concernant la quantité de protéines comprise entre 0,73 et $0,76 \mathrm{~g}$ pour l'ensemble des huîtres. La composition biochimique de la chair est significativement différente entre les mâles et les femelles; ces dernières étant plus riches en lipides $(0,30 \mathrm{~g})$ et glucides $(0,38 \mathrm{~g})$ que leurs homologues mâles.

Concernant les mesures physiologiques, aucune différence significative n'apparaît selon le sexe des huîtres.

En revanche, les fonctions de filtration et absorption montrent des différences significatives selon les stades de maturité sexuelle. La filtration est de $1,81 . \mathrm{h}^{-1}$

Tableau 2. - Composition biochimique et physiologie comparée (moyenne $\pm t \times \sigma / \sqrt{n}$ ). Analyse de covariance (poids sec) selon le stade de maturité -sexuelle et le sexe des huîtres. Les valeurs sont les moyennes par modalité lorsque l'effet de la covariable est retiré.

Proximate biochemical composition and comparison of physiological rates (mean $\pm t \times \sigma / \sqrt{n}$ ). Covariate analysis based on the maturity stage and sex of oysters. The results represent the average per modality without the covariute effect.

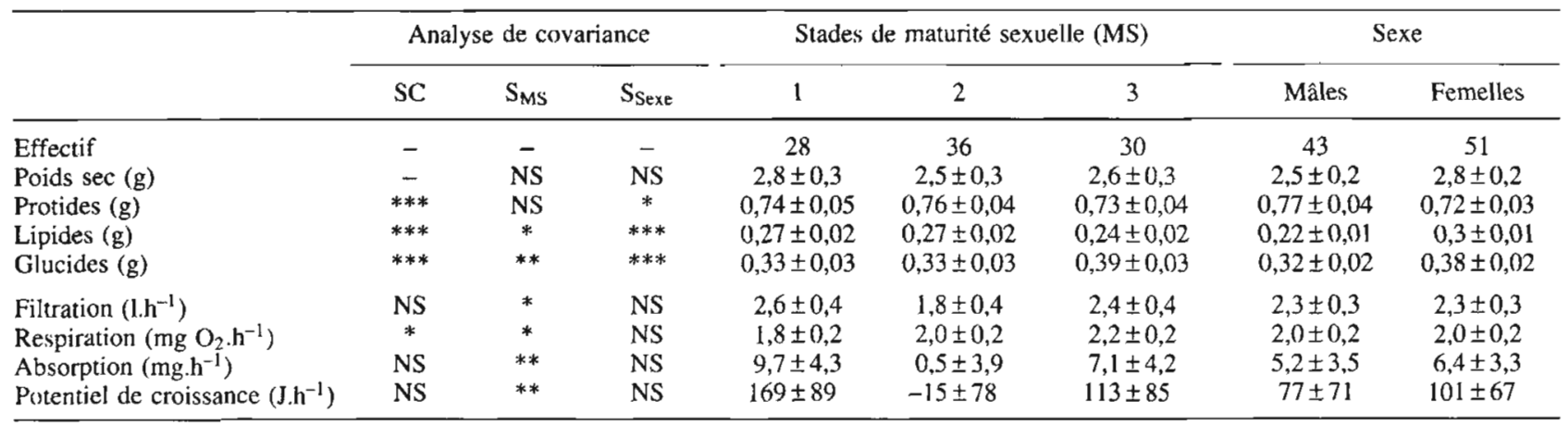

SC : Niveau de signification statistique de la covariable poids sec; $S_{M S}$ et $S_{S e x e}:$ Niveaux de signification statistique de l'effet des facteurs maturité sexuelle (MS) et Sexe, au seuil de $5 \%(*) ; 1 \%\left(^{* *}\right)$ et $1 \%(* * *)$. NS : non significatif. Stade 1 : début de gamétogenèse; stade 2 : stade de maturation avancée; stade $3:$ stade de post-ponte. 
pour le stade 2 de maturation avancée, contre 2,42,6 $1 . \mathrm{h}^{-1}$ en post-ponte et début de gamétogenèse. Cette différence entre les stades est confirméc par les résultats d'absorption et les mesures du potentiel de croissance négatif de $-15 \mathrm{~J}^{-\mathrm{h}^{-1}}$ pour le slade 2 , alors que le potentiel de croissance est positif, et compris entre 110 et $170 \mathrm{~J}^{-1} \mathrm{~h}^{-1}$ pour les stades 1 el 3 . Le test de rang de Scheffe sur les potentiels de croissance regroupe les stades de maturation $1-3$ et 2-3.

La respiration est également significativement différente entre les stades sexuels $(p=0,05)$. Elle est de $1,8 \mathrm{mg} \mathrm{O}_{2} \cdot \mathrm{h}^{-1}$ au stade 1 , de 2,0 et $2,2 \mathrm{mg} \mathrm{O}_{2} \cdot \mathrm{h}^{1}$ respectivement aux stades 2 et 3 de la maturation.

L'effet de la covariable poids sec est significatif uniquement sur la respiration $(p=0,05)$. Le test de Scheffe sur la respiration groupe les stades 1-2 et 2-3. Afin de préciser cette relation d'allométrie, un modèle de régression non linéaire est établi (Fig. 4) à partir des huîtres en cours de maturation (stades 1 et 2 ), résultats auxquels sont associés ceux obtenus au cours d'une expérimentation réalisée en 1993. Les huîtres de cette première expérimentation ont un poids moyen de 0,9 et $1,9 \mathrm{~g}$ respectivement pour les huitres aux stades de maturation 1 et 2 .

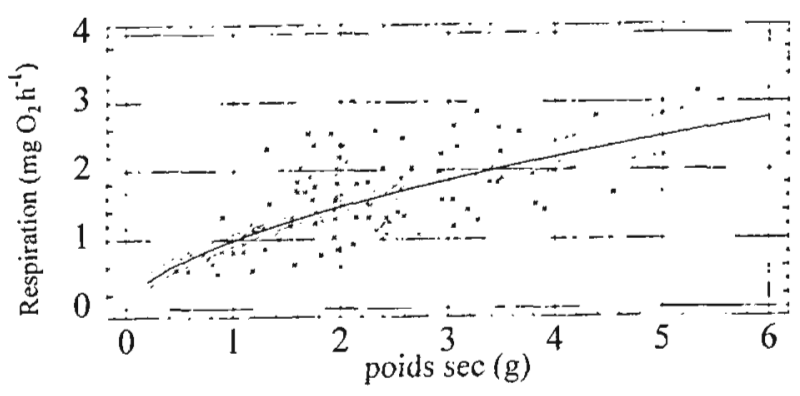

Figure 4. - Modèle du taux de respiration de l'huitre creusc $C$. gigas pendant la maturation sexuclle en fonclion du poids see de chair par individu $\left(\mathrm{mg} \mathrm{O}_{2} \mathrm{~h}^{-1}\right)$. Lignes pointillées = intervalles de confiance $d u$ modèle au seuil de $95 \%$.

Respiration rate of Crassostrea gigas daring the sexurd maturation period in funcrion of the individual oyster dry meat weight $\left(\mathrm{mg} \mathrm{O}_{2}\right.$ $h^{-1}$. Dotted lines $=55 \%$ confidence limits for the model.

A la température expérimentale de $21^{\circ} \mathrm{C}$, le modèle allométrique de respiration le mieux ajusté aux données expérimentales, est le modèle de type multiplicatif ( $p=0,001$ ) (Fig. 4). L'équation du modèle est la suivante $(n=117)$ :

Respiration $=0,99 \times \mathrm{pds}^{(0,574}$

$\mathrm{R}^{2}=0,46$ et pds : poids sec en grammes.

L'écart-type sur le coefficient d'allométrie de 0,574, est de 0,05 .

\section{Comparaison selon l'âge (1,5 et 2,5 ans) et le sexe}

Les moyennes de poids sec sont de 1,5 et $2,5 \mathrm{~g}$, respectivement pour les lots d'huîtres de 1,5 et 2,5 ans (Tableau 3). La quantité de glucides de la chair est de $0,38 \mathrm{~g}$ pour les huîtres de 1,5 ans, contre $0,28 \mathrm{~g}$ seulement pour les huitres de 2,5 ans $(p=0,001)$, alors que les teneurs des autres constituants biochimiques sont par ailleurs du même ordre de grandeur pour les 2 populations. Pour le stade de maturation 2, la teneur en lipides est significativement plus forte pour les femelles $(0,26 \mathrm{~g})$ que pour les mâles $(0,18 \mathrm{~g})$ $(p=0,0(01)$. L'effet de la covariable poids sec est significatif pour la respiration au stade 2 de maturation, comme il l'était également pour l'ensomble des 3 stades $(1,2,3)$. Aucun effet n'apparaît pour les diverses modalités des deux facteurs (âge et sexe) pour les fonctions physiologiques mesurées. Les valeurs négatives des potenticls de croissance sont en accord avec les résultats du Tableau 2 pour la population de 2,5 ans. Les huitres de 1,5 ans présentent également un potentiel de croissance négatif $\left.(-9] \mathrm{J}^{-\mathrm{h}^{-1}}\right)$ au stade 2 de la maturation.

\section{DISCUSSION}

Le cycle biochimique saisonnicr des huitres peut être utilisé comme un indicateur de leur état physiologique. Le taux maximal de glucides au cours du cycle biochimique dans le milieu naturcl, précède d'un mois la valeur maximale atteinte par les lipides au cours de la maturation (Maurer et Borel, 1986). Au début avril 1995, la teneur en glucides des huîtres cst de $16,6 \%$ pour la population d'huîtres avant le début de conditionnement. Ce taux est de 12$13 \%$ sculement pour les huîtres aux stades 1-2 de maturité sexuelle, et de $15 \%$ après la ponte. Dans les conditions de cette expérience, une température constante de $20-22^{\circ} \mathrm{C}$ permet de reproduire le cycle cle maturation en 5 à 6 semaines. Contrairement aux données du milieu naturel, aucune déplétion des réserves de glycogène n'apparaît après la ponte (stade 3). Au contraire, la quantité de glucides est même supéricure à ce stade. Ce résultat laisse supposer que dans les conditions particulières de maturation au cours de celtc expérience, les huîtres effectuent une maturation ne mobilisant qu'une partic de leurs réserves pour la formation de gamètes. L'absence de perte de poids significative des huîtres après Ia ponte, conforte cette hypothèsc. La différence significative de la tencur en lipides d'un stade de vitellogenèsc avancée au stade de post-ponte traduit la perte lipidique globale survenant lors de l'émission des produits sexuels plus riches en lipides. Les huittres femelles présentent également un taux de lipides significativement supérieur à celui des mâles (Mann, 1979; Deslous-Paoli et al., 1982). Il est d'environ 13\% pour les femelles contre 11 \% pour les mâles. Ainsi, les conditions environnementales particulièrement 
Tableau 3. - Composition biochimique et physiologie comparée (moyenne $\pm t \times \sigma / \sqrt{n}$ ). Analyse de covariance (poids sec) selon l'âge et le sexe des huitres au stade 2 de maturation. I.es valcurs sont les moyennes par modalité lorsque l'effet de la covariable est retirć.

Proximate biochemical composition and comparison of physiological rates (mean $\pm t \times \sigma / \sqrt{n}$ ). Covariate analysis based on the age and sex of oysters at stage 2 of maturation. The results represent the average per modality without the covariate effect.

\begin{tabular}{|c|c|c|c|c|c|c|c|}
\hline & \multicolumn{3}{|c|}{ Analyse de covariance } & \multicolumn{2}{|c|}{ Age } & \multicolumn{2}{|c|}{ Sexe } \\
\hline & $\mathrm{SC}$ & $S_{\text {Age }}$ & $\mathrm{S}_{\text {Sexe }}$ & 2,5 ans & 1,5 ans & Mâles & Femelles \\
\hline Poids sce (g) & - & ***** & NS & $2,5 \pm 0,3$ & $1,5 \pm 0,4$ & $1,8 \pm 0,4$ & $2.2 \pm 0.3$ \\
\hline Protides $(g)$ & $* * *$ & NS & NS & $0,66 \pm 0,04$ & $0,63 \pm 0,06$ & $0,66 \pm 0,05$ & $0,62 \pm 0,04$ \\
\hline Lipides (g) & $* * *$ & NS & $* * *$ & $0,22 \pm 0,01$ & $0,21 \pm 0,02$ & $0,18 \pm 0,02$ & $0,26 \pm 0,01$ \\
\hline Glucides (g) & $* * *$ & $* * *$ & NS & $0,28 \pm 0,02$ & $0,38 \pm 0,04$ & $0,32 \pm 0,04$ & $0,34 \pm 0,02$ \\
\hline Respiration ( $\mathrm{mg} \mathrm{O}_{2} \cdot \mathrm{h}^{-1}$ ) & $* * *$ & NS & NS & $1,8 \pm 0,2$ & $1,9 \pm 0,3$ & $1,9 \pm 0,3$ & $1,8 \pm 0,2$ \\
\hline Absorption (mg.h ${ }^{1}$ ) & NS & NS & NS & $0,4 \pm 2,4$ & $-3,2 \pm 4,4$ & $-0,8 \pm 3,7$ & $-2,0 \pm 2,6$ \\
\hline Potentiel de croissance $\left(J \cdot \mathrm{h}^{-1}\right)$ & NS & NS & NS & $-16 \pm 48$ & $-91 \pm 88$ & $-41 \pm 74$ & $-66 \pm 52$ \\
\hline
\end{tabular}

SC : Niveau de signification statistique de la covariable poids sec; $S_{\text {Age }}$ et $S_{\text {Scxe }}$ : Niveaux de signification statistique de l'effet des facteurs Agc et Sexe, au seuil de $5 \%\left({ }^{*}\right) ; 1 \%\left({ }^{* *}\right)$ et $1 \% /(x)(* *)$. NS : non significatif.

favorables au cours de ce conditionnement, induisent un cycle biochimique qui diffère sensiblement des observations faites sur les huîtres d'élevage dans le bassin de Marennes-Oléron.

Cette étude apporte une contribution significative au modèle biologique de l'huître Crassostrea gigas développé par Raillard (1991) et Barillé et al. (1997). Dans la dernière version du modèle biologique, le coefficient de 0,8 pour la respiration est appliqué aux huîtres tout au long de l'année (Bougrier et al., 1995). D'après les données de cette étude, un coefficient d'allométrie de 0,574 pourrait être utilisé en fin de période de maturation des huîtres, quand la température de l'eau est proche de $21^{\circ} \mathrm{C}$. L'allométrie est plus faible pour les huîtres en cours de maturation qu'au cours de leur croissance (hors maturité sexuelle) quand elle est égale à 0,800 (Bougrier et al., 1995) (Fig. 5). Ainsi, à poids égal, une huître au stade 2 de maturation, présente une consommation en oxygène inférieure à celle d'une huître immature. Toutefois, les besoins énergétiques de respiration augmentent significativement avec la prise de poids liée à la maturation de l'huître, ce qui n'est pas le cas pour la fonction de filtration. La prise de poids liée au développement des gonades ne s'accompagne pas d'une évolution anatomique de la branchie, ni d'un changement de rythme de filtration. En revanche, le maintien de la fonction de filtration à son niveau d'activité de stade immature pourrait contribuer à affaiblir l'animal, quand la demande en oxygène augmente, consécutivement à la prise de poids liée à la maturation. Ce résultat pourrait révéler un stress physiologique qui doit être mis en relation avec les mortalités estivales observées ponctuellement sur le bassin de Marennes-Oléron. Ainsi, quand le poids de . l'huître passe de 1,25 à $2,65 \mathrm{~g}$ au cours du mois de conditionnement, la consommation d'oxygène passe de 0,8 à $1,8 \mathrm{mg} \cdot \mathrm{h}^{-1}$, correspondant en fait à une demande énergétique de 15 joules par heure. Mais cette dépense énergétique "supplémentaire » reste faible devant les différences de potentiel de croissance de 100-200 J.h $\mathrm{h}^{-1}$ notées entre les huîtres au stade sexuel 2 et celles à un stade sexuel de début de maturation (1) ou de post-ponte (3). Le potentiel de croissance négatif pour le stade 2 de maturation sexuelle avancée, est un résultat essentiel de cette étude. Davis (1988) dans une comparaison entre triploïdes et diplö̈des de Crassostrea gigas en période de reproduction montre que les huîtres diploïdes sous condition «stressante " liée à la maturation peuvent présenter un bilan énergétique négatif. Thompson (1984) et Newell et Thompson (1984) notent une réduction significative de l'activité de filtration chez la moule lors de sa période hivernale de ponte, mais avec un bilan énergétique qui reste positif. Dans le cas de notre étude, cette différence de potentiel de croissance entre les stades de maturation, dans des conditions alimentaires « améliorées » par rapport aux conditions du milieu naturel, ne peut être expliquée par la seule

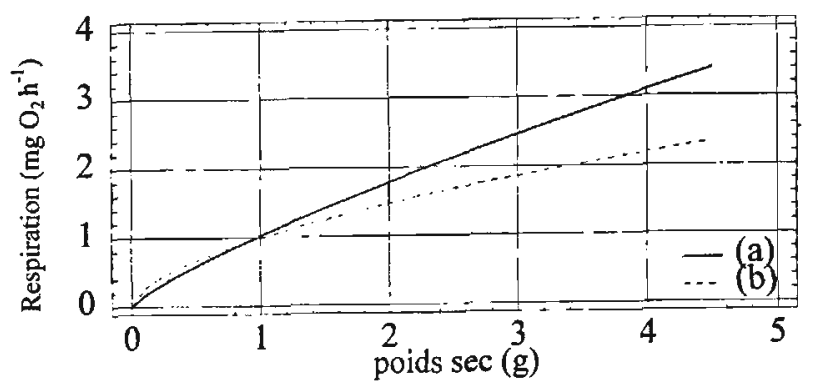

Figure 5. - Comparaison des modèles de respiration de l'huître creuse $C$. gigas en période de repos sexuel (a) et en période de maturation sexuelle (b). (a) : Respiration $=1,022 \times(\text { poids sec })^{0,800}$; (b) : Respiration $=0,987 \times(\text { poids sec })^{0,574}$.

Comparison of respiration rate models for the cupped oyster Crassostrea gigas during resting period $(a)$ and sexual maturation $(b)$. (a): Respiration $=1.022 \times(\text { dry weight })^{0.800} ;(b):$ Respiration $=0.98 \times(d r y$ weight $)^{0,574}$. 
baisse de l'activité de filtration de 2,6 à 1,8 l.h . Seule une chute importante de l'activité d'absorption mesurée entre le stade 2 et les autres stades peut conduire à ce résultat. L'importance de l'intervalle de confiance autour des valeurs d'absorption montre combien la variabilité individuelle est grande pour un même stade de maturité sexuelle.

Dans le cadre de cette étude, aucune différence significative n'a été mise en évidence entre les deux populations de 1,5 et 2,5 ans à un stade de maturation avancée (stade 2). Par contre, les réponses physiologiques obtenues pour le stade 2 avec la population d'huîtres de 1,5 ans, confortent et confirment les résultats issus de la première analyse avec la classe d'âge de 2,5 ans. Des études ultérieures pourraient comparer ces deux classes d'âge aux autres stades de maturité, et prendre en compte une classe d'âge encore plus jeune.

Parmi les résultats, il faut noter également l'efficacité de la méthodologie de conditionnement qui permet de disposer simultanément des principaux stades de maturation rencontrés dans le milicu naturel. Cette méthodologie peut être utilisée dans des expériences in situ faisant appel à des indicateurs biologiques à différents stades de maturité sexuclle. Malgré des conditions alimentaires, en laboratoire, nettement plus favorables pour les huîtres que celles rencontrées dans le milieu naturel, cette étude révèle un potentiel de croissance négatif de $C$. gigas durant la phase de maturation avancéc, alors que ce potentiel de croissance est positif pour les autres stades. Cette relation entre le potenticl de croissance et la maturation doit être précisée au cours d'études ultérieures. Ainsi, dans un contexte environnemental défavorable, de milieu surexploité et de ressources trophiques faibles, les populations d'huîtres à un stade de maturation avancée pourraient présenter un terrain propice à l'expression d'un agent pathogène ou à tout autre facteur de stress, et engendrer ainsi des mortalités printanières ou estivales. Des études ultérieures pourraient préciser la distribution des stades de maturation de Crassostrea gigas sur les différents bancs ostréicoles du bassin de Marennes-Oléron, afin de connaître l'état physiologique des huîtres en cas d'épisodes de mortalité survenant sur les cheptels.

\section{RÉFÉRENCES}

Bacher C. 1989. Capacité trophique du bassin de MarennesOléron : couplage d'un modèle de transport particulaire et d'un modèle de croissance de l'huitre Crassostrea gigas. Aquat. Living Resour. 2, 199-214.

Barillé L., J. Prou, M. Héral, S. Bougrier 1993. No influence of food quality, but ration- dependent retention efficiencies in the Japanese oyster Crassostrea gigas. J. Exp. Mar. Biol. Ecol. 171, 91-106.

Barillé L., M. Héral, A.L. Barillé-Boyer 1997. Modélisation de l'écophysiologie de l'huître Crassostrea gigas dans un environnement estuarien. Aquat. Living Resour. 10, 31-48.
Bayne B. L. 1976. Marine musscls: their ecology and physiology. B. L. Bayne eds. Cambridge University Press Publisher, Australia, $506 \mathrm{p}$.

Bayne B. L., D. A. Brown, K. Burns, D. R. Dixon, A. Ivanovici, D. R. Linvingstone, D. M. Lowc, M. N. Moore, A. R. D. Stebbing, J. Widdows 1985. The effect of stress and pollution on marine animals. Praeger Publishing Co., $384 \mathrm{p}$.

Bcrthomé J. P., J. M. Deslous-Paoli, M. Héral, D. Razet, J. Garnier 1982. Absence de captage de Crassostrea gigas dans le bassin de Marennes-Oléron en 1981 : causes et conséquences. Cons. Int. Explor. Mer. E, 26 p.

Bodoy A., J. Garnier, D. Razet, P. Geairon 1990). Mass mortality of oysters, Crassostrea gigas during spring 1988 in the bay of Marennes-Oléron, related to environmental conditions. Cons. Int. Explor. Mer. K 11, 23 p.

Bougrier S., P. Geairon, J. M. Deslous-Paoli, C. Bacher, G. Jonquières 1995. Allometric relationships and effects of temperature on clearance and oxygen consumption rates of Crassostrea gigas (Thunberg). Aquaculture 134, 143-154.

Davis J. P. 1988. Energetic of sterile triploid nysters uncouple the reproductive and somatic effort of diploids. J. Shellfish Res. 7, 114.

Deslous-Paoli J. M., M. Héral, J. P. Berthomé, D. Razet, J. Garnier 1982. Reproduction naturelle de Crassostrea gigas (Thunberg) dans le bassin dc Marennes-Oléron en 1979 et 1981 : aspect biochimique et énergétiques. Rev. Trav. Inst. Pêches Marit. 45, 319-327.

Deslous-Paoli J. M., J. M. Sornin, M. Héral 1987. Variations saisonnières in situ de la production et de la composition des biodépôts de trois mollusques estuariens (Mytilus edulis, Crassostrea gigas, Crepidula fornicata). Haliotis 16, 233-245.

Deslous-Paoli J. M., M. Héral 1988. Biochemical composition and energy value of Crassostrea gigas (Thunberg) cultured in the bay of Marennes-Olćron. Aquat. Living Resour. 1, 239-249.

Deslous-Paoli J. M., A. M. Lannou, P. Geairon, S. Bougricr, O. Raillard, M. Héral 1992. Effect of feeding bchaviour of Crassostrea gigas (bivalve molluscs) on biosedimentation of natural particulate matter. Hydrobiologia 231, 85-91.

Dinamini P. 1987. Gamctogenetic pattern in populations of Pacific oyster, Crassostrea gigas, in Northland, New Zealand. Aquaculture 64, 65-76.

Gabbott P. A. 1976. Energy metabolism. In: Marine mussels. Their ecology and physiology. Bayne ed. Cambridge Univ. Press, 293-355.

Héral M., J. M. Deslous-Paoli, J. M. Somin 1983. Transferts énergétiques entre l'huître $C$. gigas de 1 an et la nourriture potenticlle disponible dans un bassin ostréicole : premières approches. Océanis 9, 169-194.

Le Dantec J. 1968. Écologie et reproduction de l'huître portugaise ( $C$. angulata) dans le bassin d'Arcachon et la rive gauche de la Gironde. Rev. Trav. Inst. Pêches Marit. 32, 237-262.

Lubet P. 1959. Recherche sur le cycle sexuel et l'émission de gamètes chez les Mytilidae et les Pectinidae (Mollusques Bivalves). Rev. Trav. Off. Pêches Marit. 23, 387-548.

Lubet P. 1991. Bases biologiques de la culture des mollusques. In: Bases biologiques et écologiques de l'aquaculture. Lavoisier, Tech. et Doc., 500 p.

Lucas A. 1982. La nutrition des larves de bivalves. Océanis 8, 363-388. 
Mann R. 1979. Some biochemical and physiological aspects of growth and gametogenesis in Crassostrea gigas and Ostrea edulis grown at sustained elevated temperatures. J. Mar. Biol. Assoc. U.K. 52, 95-110.

Marteil L. 1976. La conchyliculture française, $2^{\mathrm{e}}$ partie. Biologie de l'huître et de la moule. Rev. Trav. Inst. Pêches Marit. 40, 149-346.

Maurer D., M. Comps, H. His 1986. Caractéristiques des mortalités estivales de l'huître Crassostrea gigas dans le bassin d'Arcachon. Haliotis 15, 309-317.

Maurer D., M. Borel 1986. Croissance, engraissement et cycle sexucl de Crassostrea gigas dans le bassin d'Arcachon. Comparaison des huîtres âgées de 1 et 2 ans. Haliotis 15, 125-134.

Mori K. 1979. Effects of artificial eutrophication on the metabolism of the Japanese oyster Crassostrea gigas. Mar. Biol. 53, 361-369.

Newell R. I. E., R. J. Thompson 1984. Reduced clearance rates associated with spawning in the mussel, Mytilus edulis (L.) (Bivalvia, Mytilidac). Mar. Biol. Lett. 5, 21-33.

Perdue J., J. H. Beattie, K. K. Chew 1981. Some relationship between gametogenic cycle and summer mortality phenomenon in the Pacific oyster (Crassostrea gigas) in Washington State. J. Shellfish Res. 1, 9-16.
Price K., D. Maurer 1971. Holding and spawning Delaware Bay oysters (Crassostrea virginica) out of season. 2 Temperature requirement for maturation of gonads. Proc. Natl. Shellfish Assoc. 61, 29-34.

Raillard O. 1991. Etude des l'interactions entre les processus physiques et biologiques intervenant dans la production de l'huître Crassostrea gigas (Thunberg) du bassin de Marennes-Oléron : essai de modélisation. Thèse dr Univ. Paris VI, 212 p.

Razet D., M. Héral, J. Prou , J. Legrand, J. M. Sornin 1990. Variation des productions saisonnières de biodépôts (fèces ct pseudofèces) de l'huître Crassostrea gigas dans un estuaire macrotidal : baie de Marennes Oléron. Haliotis 10, 143-161.

Shpigel M., B. J. Barber, R. Mann 1992. Effects of elevated temperature on growth, gametogenesis, physiology, and biochemical composition in diploid and triploid Pacific oysters, Crassostrea gigas Thunberg. J. Exp. Mar. Biol. Ecol. 151, 15-25.

Thompson R. J. 1984. The reproductive cycle and physiological ecology of the mussel Mytilus edulis in a subarctic, non estuarine environment. Mar. Biol. 79, 277-288. 